Research Article

\title{
Structure Design and Analysis of Canted-Cosine-Theta (CCT) Superconducting Quadrupole Magnet
}

\author{
Luncai Zhou $\mathbb{D}^{1,},{ }^{1,2}$ Rongzhen Zhao $\mathbb{D}^{1},{ }^{1}$ Yuquan Chen $\mathbb{D}^{\circ},{ }^{2}$ Liang $Y u\left(\mathbb{D},{ }^{2}\right.$ Wei Wu $\mathbb{D}^{\circ},{ }^{2}$ \\ and Lizhen $\mathrm{Ma} \mathbb{D D}^{2}$ \\ ${ }^{1}$ Lanzhou University of Technology, Lanzhou 730050, China \\ ${ }^{2}$ Institute of Modern Physics, Chinese Academy of Sciences, Lanzhou 730000, China \\ Correspondence should be addressed to Rongzhen Zhao; 986728134@qq.com
}

Received 11 June 2020; Revised 23 January 2021; Accepted 24 May 2021; Published 1 June 2021

Academic Editor: Selda Oterkus

Copyright (C) 2021 Luncai Zhou et al. This is an open access article distributed under the Creative Commons Attribution License, which permits unrestricted use, distribution, and reproduction in any medium, provided the original work is properly cited.

\begin{abstract}
A superconducting quadrupole magnet based on the Canted-Cosine-Theta (CCT) type coil with a gradient field of $40 \mathrm{~T} / \mathrm{m}$ and a bore diameter of $60 \mathrm{~mm}$ has been designed for the preresearch of two projects of high intensity accelerator facility (HIAF) and accelerator driven subcritical system (ADS). The magnet is comprised of two-layer coils embedded in the formers and the end plates for locating. The coil formers made of aluminum alloy are machined with grooves according to the drive equations of CCT for placing the wires. The existence of ribs between two adjacent wires can avoid accumulation of the electromagnetic force. It is important to take the mechanical design for the complex structure to avoid tensile stresses on the conductor and confine the stresses within a reasonable value. The stress analysis for the quadrupole magnet has been carried out considering the thermal shrinking due to cool down as well as the electromagnetic force on the coil. This paper reports the detailed stress analysis for the CCT quadrupole magnet structure, discusses the calculating results, and gives a reasonable mechanical design.
\end{abstract}

\section{Introduction}

High intensity accelerator facility (HIAF) and accelerator driven subcritical system (ADS) are under construction at the Institute of Modern Physics in China [1, 2]. HIAF is a new project to pursue nuclear physics research which consists of a booster ring called BRing, a spectrometer ring called SRing, and a fragmentation separator called HFRS. And, HFRS is an important connection between BRing and SRing with the field rigidity of $25 \mathrm{~T} \cdot \mathrm{m}$ and a big beam acceptance of $\pm 160 \mathrm{~mm}$. To meet the requirements of the magnetic field, the CCT superconducting magnets are presented because it can produce a good field with smaller cold mass than the superferric-type magnet [3]. ADS is the most promising device for the safe treatment of long-life nuclear waste which consists of a superconducting proton linac, a high-power spallation target, and a subcritical reactor. In the superconducting linac, the solenoids are used heavily for beam focus. In order to increase the magnetic field gradient further, the CCT quadrupole magnet was proposed to explore the possibility for employing at the superconducting linac. It would be installed in the same cryostat with the superconducting cavities so as to shorten the total length and save cost [4].

In order to verify the feasibility for real application, a CCT superconducting quadrupole magnet prototype was developed. It has a bore diameter of $60 \mathrm{~mm}$ and efficient length of $160 \mathrm{~mm}$. For higher efficiency, ten wires were placed in one groove by the $2 \times 5$ arrangement with series connection. The operating current is $400 \mathrm{~A}$. The stability of superconducting magnet mechanical structure is one of the main reasons for quench [5-8], so the stress-strain analysis on the magnet structure plays an important role on the magnet design [9-15], particularly for the complex structure of the CCT quadrupole magnet. The analysis model is created by cutting a section from the structure with a pitch owing to its periodic symmetry in the axial direction. Based on the 3D model, the stress analysis was carried out in the ANSYS software considering the thermal strain during cooling down and the electromagnetic force at the operating 
current of $400 \mathrm{~A}$. The surfaces between the support structure and the coil are in contact during the operation, and the contact analysis model is used to make the results closer to the real situation. The results show that the low-field CCT superconducting quadrupole magnet can meet the rigidity requirements of the magnet by strengthening the internal structure without external support structure.

\section{Mechanical Structure}

The mechanical structure of the CCT quadrupole is depended much on the coil form with the conductor path according to the following equations. As a result, the current flow lines are distributed on the surface of a cylinder and bring about a $\cos 2 \theta$ like current distribution in the axial direction. However, beside axial current, there is azimuthal current component which can produce axial field. In order to attain a pure quadrupole field, two superimposed coils with oppositely current direction are used to cancel the axial field (see Figure 1). It can be seen that the winding of each layer exhibits a periodic symmetry in the axial direction, which also can be derived by equations (1)-(3). The minimum periodic length is the pitch of the conductor path which is the turn-to-turn axial distance expressed by $h$. The symmetry region can be repeated in a laminated pattern to form the complete CCT layer [12]:

$$
\begin{aligned}
& x(\theta)=R \cdot \cos (\theta), \\
& y(\theta)=R \times \cos (\theta), \\
& z(\theta)=\frac{h}{2 \pi} \theta+\sin (2 \cdot \theta) \cdot \cot (\alpha) .
\end{aligned}
$$

For the accuracy of coil positioning and the feasibility of winding, also considering the effect of electromagnetic force, the best choice of the coil mandrels are some cylindrical tubes with grooves on its surfaces, as shown in Figure 2. The grooves are machined out of the tube and the remaining part is the mandrel which is comprised of the ribs and cylindrical spar. The ribs located between turns can intercept the accumulation of Lorentz force and transfer them to the spar. The spars and ribs provide structural support for the CCT coil. The shapes of the grooves and ribs agree with the quadrupole winding which is shown in Figure 1. Therefore, the mandrel is also axially periodic and so does the whole magnet structure.

Figure 3 shows the assembly of the quadrupole prototype which consists of two-layer mandrels, two end plates, positioning keys, and an outer aluminum shell. Table 1 lists the main parameters of the quadrupole structure. The positions of the mandrels and the cylinders are limited in the circumferential direction by using the keys so as to ensure the circumferential installation accuracy. And, the axial assembling accuracy is fulfilled by the end plates with locating pins on it.

\section{Structure Analysis}

3.1. FEM Model. The quadrupole prototype includes two conductor layers; with each layer, ten wires are positioned into one groove together, as shown in Figure 4. The diameter of the superconducting $\mathrm{NbTi}$ wire is $1 \mathrm{~mm}$ and the cross section of the groove is $2 \times 5 \mathrm{~mm}^{2}$. The ten conductors in the coil channel are treated as a rectangular region for model simplification. In fact, the coil cross-section perpendicular to the conductor path is actually a rectangular area after being impregnated.

Having the benefit of the periodic symmetry for the CCT structure, a 3D magnet model with a single symmetry region can be created for the structure analysis. The analysis model created by ANSYS software [16] is depicted in Figure 5. The axial length is just one pitch. The interfaces between the conductors and the mandrel are treated as bonded because the void space in the channels are filled by epoxy resins. The displacements on the contact surface between the two layers are coupled together by using the CPINTF command. To satisfy the requirement for periodic symmetry characteristic, the meshes on the two axial faces should be coincident. One of the axial faces is meshed first and then the meshes are copied into the other axial face. Therefore, the nodes on the two surfaces are matched. The element type of SOLID186 is used for the structural calculation and all of the meshes are hexahedrons.

3.2. Boundary Conditions. The generalized plane strain condition is achieved by setting the difference between the node displacements on the axially constrained surface $\left(\delta_{\mathrm{z}}=0\right)$ to zero. The axial length of the CCT model will change during the cold shrinking process, so generalized strain conditions are not suitable. For the demand of periodic axial symmetry, the matching nodes on the two axial surfaces coupled their transverse displacement and related the axial displacement to a constant length $\delta_{z}$. In this analysis, we use the second condition in $[13,15]$ which is closest to the behavior of CCT with $\delta_{z} \neq 0$ for all constrained nodes taking thermal contraction into account. The value of $\delta_{z}$ is defined as the net force over the entire magnet symmetry surface is zero. It can be determined by calculating the total force on the outer axial faces in ANSYS with two assumed $\delta_{z}$ value and to interpolate linearly the zero net force value. Figure 6 shows the interpolating process for attaining the correct $\delta_{z}$ value.

3.3. Lorentz Force. The Lorentz force on the conductors is significant for the superconducting magnet with large operating current and high field. The forces are calculating in Opera3D with the CCT coil model created by a series of 20node brick conductors. The design parameters of the quadrupole magnet are listed in Table 2.

The centroid coordinates of the elements for the current-carrying conductors are exported from ANSYS as a table file, and then, the Lorentz force density expressed by $J \times B$ at these coordinates are calculating in Opera3D [17] by processing the table file. The Lorentz force density on the conductors is shown in Figure 7 . The maximum value is $7.8 \times 10^{8} \mathrm{~N} / \mathrm{m}^{3}$. Then, the force on each element can be obtained by multiplying the corresponding element volume (4). Finally, the element force should be shared on all of its nodes and the Lorentz forces for loading on each node are prepared. 


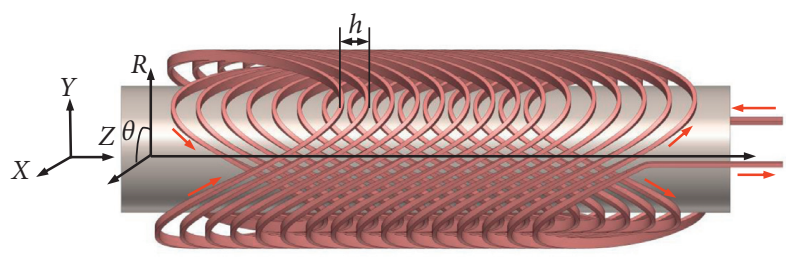

FIgUre 1: Conceptual view of CCT quadrupole winding.

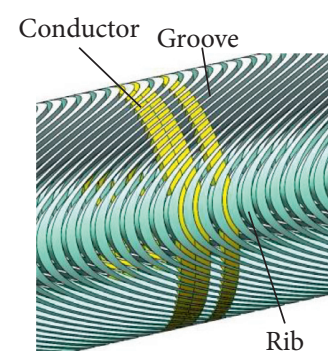

(a)

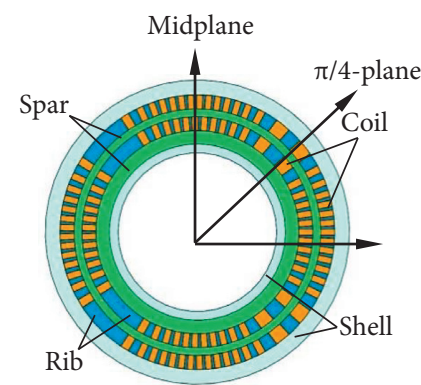

(b)

FIGURE 2: Mechanical drawing of quadrupole former with partial conductors and the cross section of the magnet.

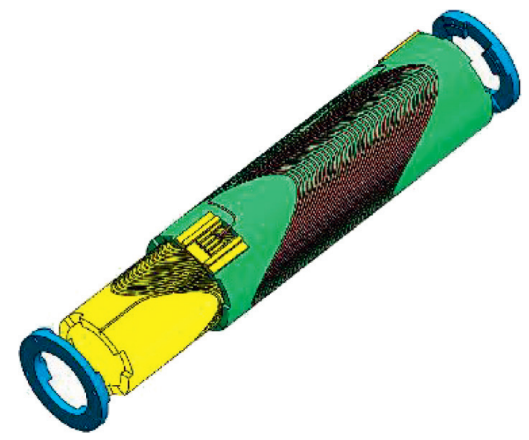

FIgURE 3: Assembly of the CCT quadrupole prototype.

TABle 1: Parameters of the quadrupole magnet structure.

\begin{tabular}{lcc}
\hline Parameters & Unit & Values \\
\hline Diameter of inner and outer layer mandrel & $\mathrm{mm}$ & $60 / 79$ \\
Thickness of inner and outer spar & $\mathrm{mm}$ & $3 / 2.6$ \\
Distance between two mandrels & $\mathrm{mm}$ & 0.2 \\
Minimum rib thickness & $\mathrm{mm}$ & 0.61 \\
Winding pitch & $\mathrm{mm}$ & 6 \\
Tilted angle & $\mathrm{deg}$ & 45 \\
Groove size & $\mathrm{mm}^{2}$ & $2 \times 5$ \\
Thickness of end plate & $\mathrm{mm}$ & 16 \\
\hline
\end{tabular}

$$
\left(\begin{array}{c}
F_{x} \\
F_{y} \\
F_{z}
\end{array}\right)=\left(\begin{array}{cc}
J_{y} & J_{z} \\
J_{z} & J_{x} \\
J_{x} & J_{y}
\end{array}\right)\left(\begin{array}{ccc}
B_{z} & B_{x} & B_{y} \\
-B_{y} & -B_{z} & -B_{x}
\end{array}\right) \times V
$$

3.4. ANSYS Calculation. The 3D finite element model with periodic axial symmetry for CCT quadrupole has been established and the Lorentz force load has also been calculated. The material of coil mandrel is aluminum alloy and the impregnated coil is treated as composites of $\mathrm{NbTi} / \mathrm{Cu}$ wire and epoxy resin. The effective Yong's modulus is used for the coil based on the homogenization method. The material properties of coil and mandrel are listed in Table 3.

The thermal contraction from room temperature to $4.2 \mathrm{~K}$ and Lorentz force is taken as loads onto the model for the structure analysis. The winding tension is not considered.

\section{Results and Discussion}

4.1. Stress Analysis with Lorentz Force. It starts to research the deformation and stress of the CCT quadrupole magnet which sustained only the Lorentz force. One of the advantages of CCT type magnet is that the electromagnetic force can be separated by ribs in the axial direction and prevented from accumulating. Figure 8 shows the displacement and stress of the coil under the effect of Lorentz force. It can be seen that the deformations feature the rotational symmetry in the circumferential direction with an angle of $90 \mathrm{deg}$ which is in according with the mechanical characteristic of the quadrupole magnet. The 


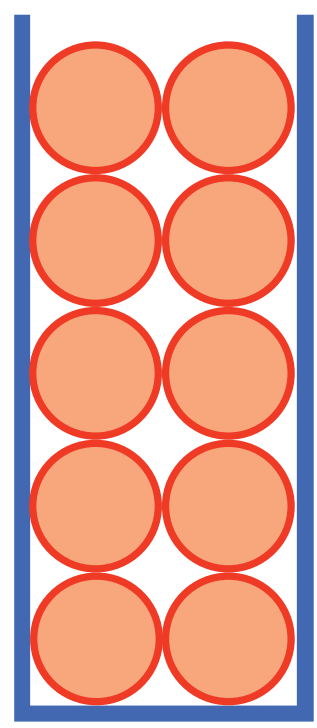

FIGURE 4: Schematic diagram of conductor placement in grooves for each layer.

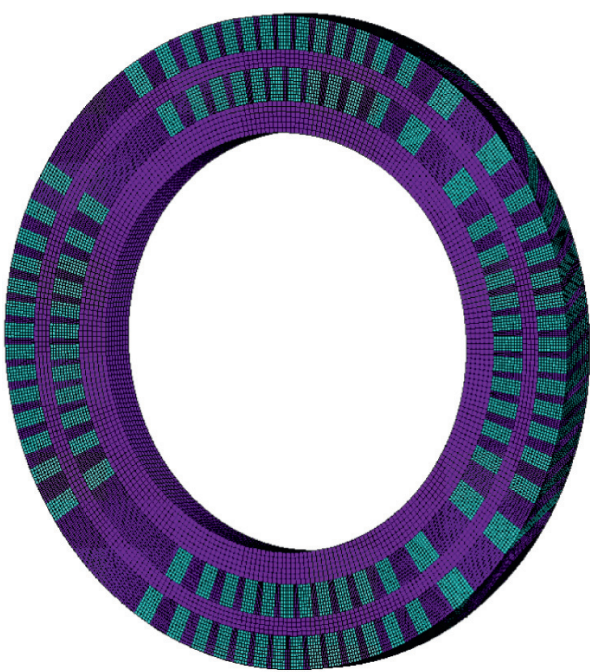

FIGURE 5: The 3D periodic symmetry model for structural analysis in ANSYS for CCT quadrupole with two layers.

conductors are pulled in the middle plane and pressed in the $\pi / 4$-plane (seen in Figure 2).

The maximum field on the conductor is about 1.9 Tesla with the operating current of $400 \mathrm{~A}$. The calculation shows the maximum displacement of the coil is 0.05 microns at the location of midplanes. The equivalent stress on the conductors is less than $10 \mathrm{MPa}$. The results indicate that the outer shell may be not necessary in this quadrupole magnet according to the analysis results because the electromagnetic force is not very large with the low magnetic field. It is however better to assemble an outer shell to restrict the conductor deformation and resist the Lorentz force especially for the high-field CCT magnet.

4.2. Stress Analysis with Loads of Thermal Contraction and Lorentz Force. The thermal strain is indeed unignored due to the large temperature difference and the inconsistent

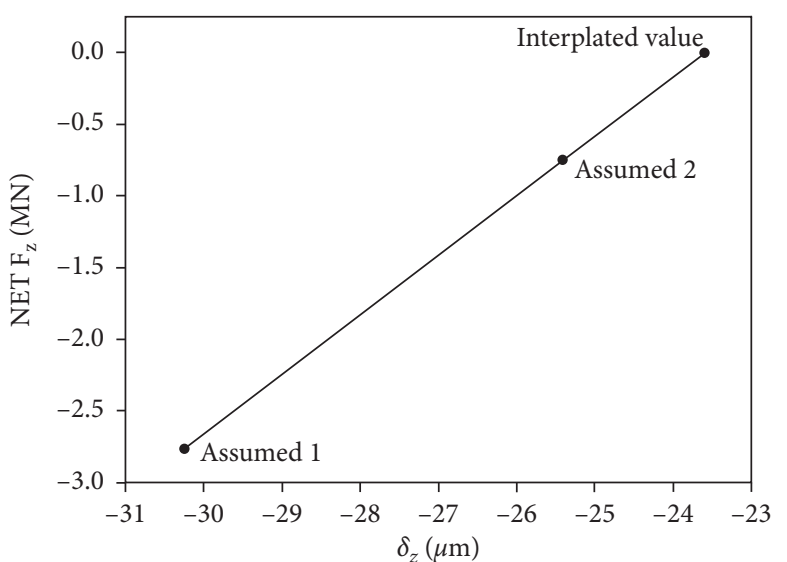

FIgURE 6: The interpolating process of zero net force for $\delta_{z}$ by two assumed values.

TABLE 2: The main parameters of magnets.

\begin{tabular}{lc}
\hline Parameters & Value \\
\hline Magnetic field gradient & $40 \mathrm{~T} / \mathrm{m}$ \\
Inclination angle & $45 \mathrm{deg}$ \\
Wires in groove & $5 \times 2$ \\
Coil layer & 2 \\
Turns per layer & 28 \\
Operating current & $400 \mathrm{~A}$ \\
\hline
\end{tabular}

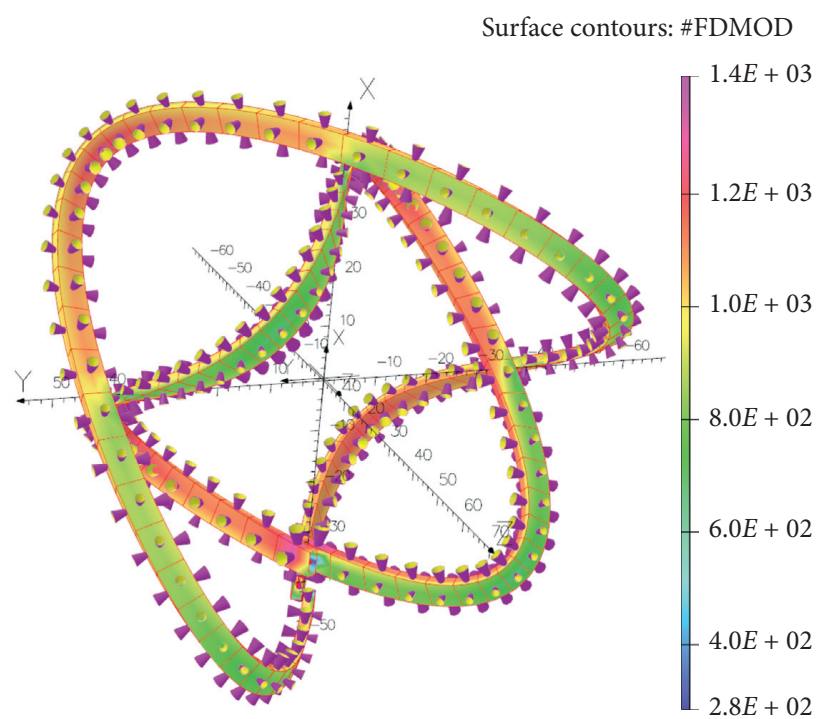

FIGURE 7: The distribution of the Lorentz force density calculated in Opera3D by $J \times B$.

shrinkage coefficient of the structural materials. And, the larger the structure scale is, the severer the thermal contraction. The stress analysis is taken step by step which is responding to the actual process of cooling and energizing. Two points on the inner surface are constrained to restrain the moving of the rigid body.

Figure 9 shows the radial displacement and equivalent stress distributions of the structure during cooling down from $293 \mathrm{~K}$ to $4.2 \mathrm{~K}$. The results show that it shrinks inward 
TABLE 3: Material properties for CCT quadrupole structure analysis.

\begin{tabular}{lccr}
\hline Materials & Elastic modulus $(\mathrm{GPa})$ & Poisson's ratio & Thermal expansion coefficient \\
\hline Coil (composites of $\mathrm{NbTi} / \mathrm{Cu}$ and resin) & 80 & 0.3 & $10.5 \times 10^{-6}$ \\
Al6061 & 70 & 0.3 & $14.3 \times 10^{-6}$ \\
\hline
\end{tabular}

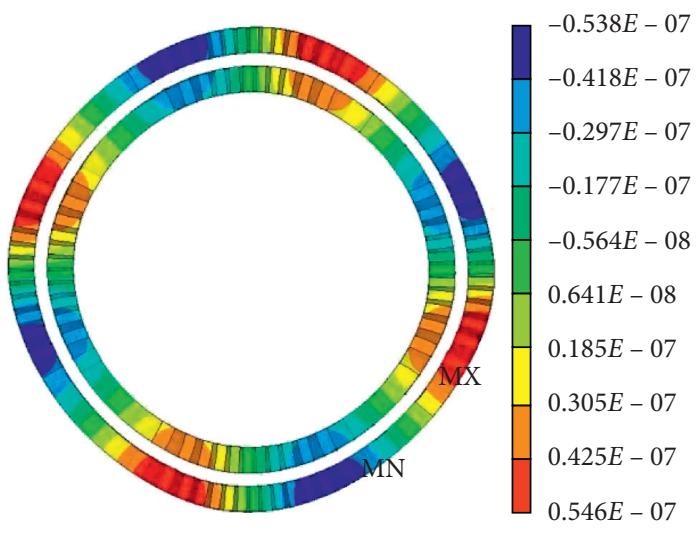

(a)

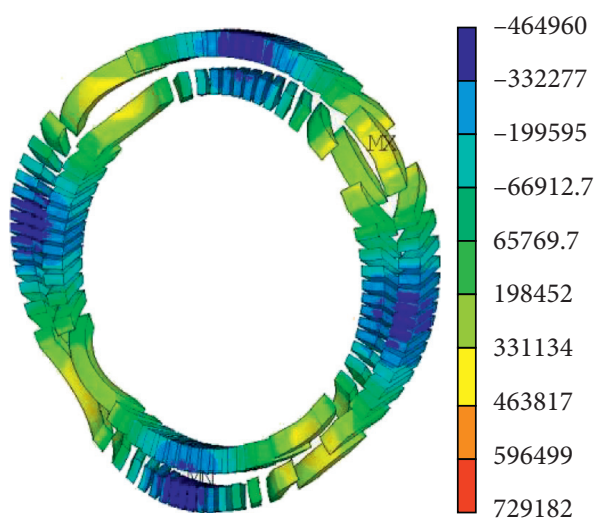

(b)

Figure 8: The displacement (a) and equivalent stress (b) distributions of the CCT quadrupole magnet under the Lorentz force.

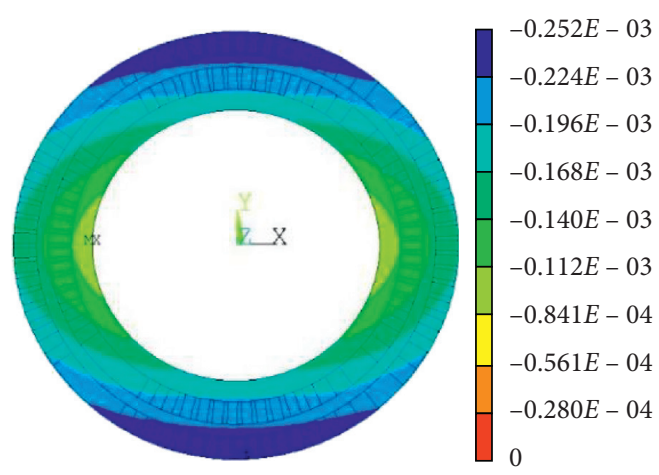

(a)
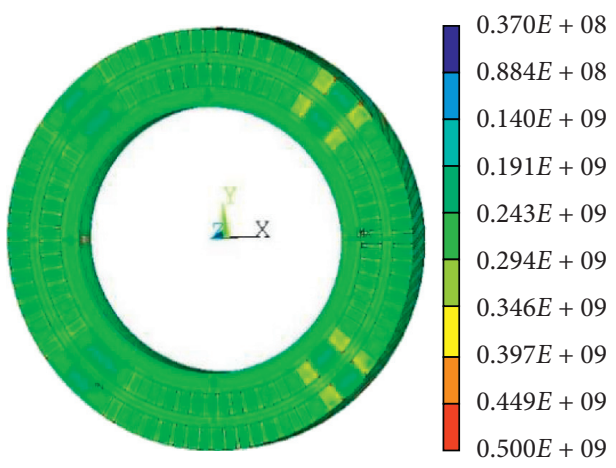

(b)

FIgURE 9: The radial displacement distribution (a) and equivalent stress distribution (b) of the structure during cooling down.

by a maximum of $0.25 \mathrm{~mm}$ which is agreed with the thermal shrinkage coefficient of the material of aluminum. The stress concentrated at the location of the constraint points however is not real. The most part of the structure is below $300 \mathrm{MPa}$. The stresses at the contact region between the conductors and ribs are a little larger which is up to $500 \mathrm{MPa}$. It results from the identical deformation on the nodes of these contact areas by gluing all of the ribs and conductors.

Figure 10 presents the radial and azimuthal stresses which are distributed on the conductors. It can be seen that the radial stresses are within $100 \mathrm{MPa}$ which are compressed in the majority of the conductors, and the azimuthal stresses are mostly less than $150 \mathrm{MPa}$. However, the stresses are larger at the shared edge which is located at the pi/4 plane and its folded symmetry position. These locations are also corresponding with the magnet poles in which the conductors are like an arch shape. It can be eliminated by not only taking a contact analysis but also increasing the complexity of creation of the contact pairs and the finite element model.

Then, the Lorentz forces are loaded to the conductors in the second step of the structural analysis. The forces are calculated in OPERA software with the operating current of $400 \mathrm{~A}$. Figure 11 shows the stresses on the conductors which is almost not different with the results of thermal contraction because of the smaller displacement under the Lorentz force with respect to thermal shrinking.

The maximum and minimum conductor stresses in the cylindrical coordinate system are shown in Table 4 . The highest stress for the conductor is found in the innermost layer. This value is $141 \mathrm{MPa}$ in the inner coil.

Figure 12 presents the equivalent stress on the spars and ribs of the mandrels. The most part of the ribs are subjected to the stress of $300 \mathrm{MPa}$ which is also below the yield strength of the Al-6061. 


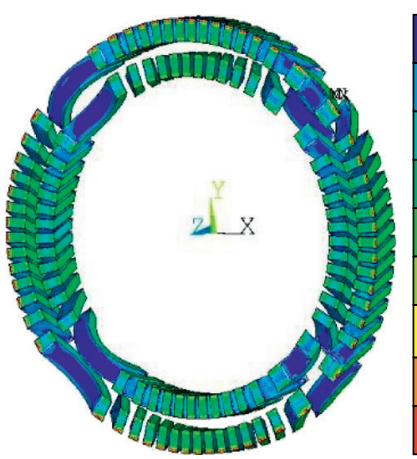

(a)
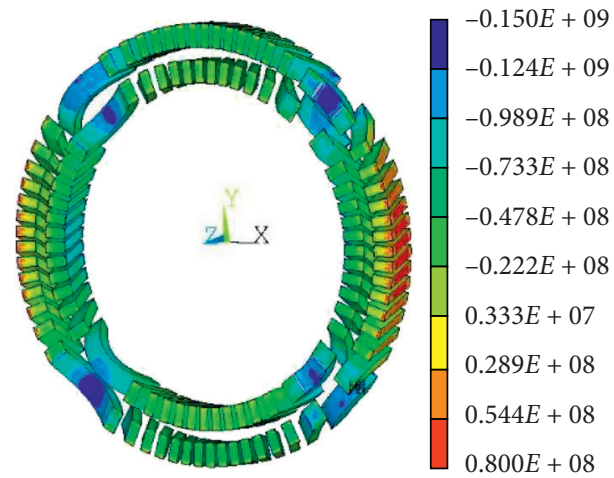

(b)

Figure 10: The radial (a) and azimuthal (b) stress distributions on the conductors when cooled down to $4.2 \mathrm{~K}$.

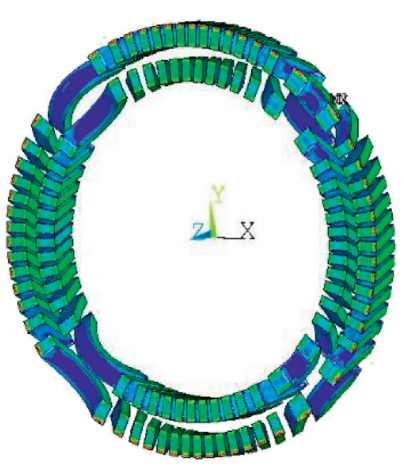

(a)

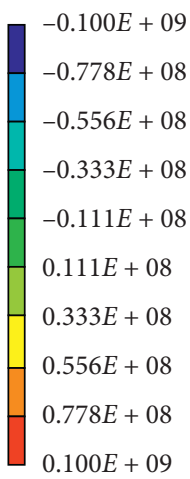

$0.100 E+09$
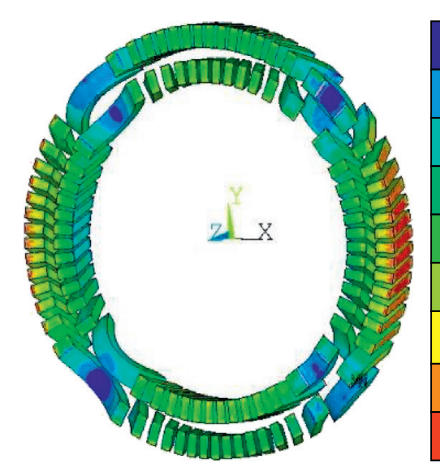

$-0.150 E+09$
$-0.124 E+09$
$-0.989 E+08$
$-0.733 E+08$
$-0.478 E+08$
$-0.222 E+08$
$0.333 E+07$
$0.289 E+08$
$0.544 E+08$
$0.800 E+08$

(b)

FiguRE 11: The radial (a) and azimuthal (b) stress distributions on the conductors with loads of thermal contraction and Lorentz force.

TABLE 4: The stress of conductor (in MPa).

\begin{tabular}{llcr}
\hline & & Cooldown to $4.2 \mathrm{~K}$ & Operation at $0.4 \mathrm{kA}$ \\
\hline \multirow{3}{*}{ Inner coil } & $\sigma_{r}$ & $-90 / 10$ & $-85 / 10$ \\
& $\sigma_{\theta}$ & $62 / 136$ & $54 / 141$ \\
\multirow{3}{*}{ Outer coil } & $\sigma_{z}$ & $-85 / 16$ & $-80 / 20$ \\
& $\sigma_{r}$ & $-78 / 28$ & $-72 / 20$ \\
& $\sigma_{\theta}$ & $23 / 103$ & $22 / 133$ \\
\hline
\end{tabular}

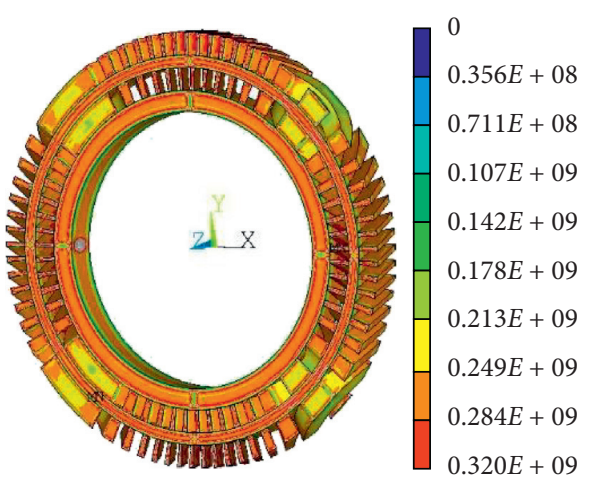

FIGURE 12: The equivalent stress on the magnet mandrels. 
In fact, the method, which imposes the identical displacement on the interface between the conductors and the mandrel, will bring about a larger stress than the truth value. So, it can be regarded as a conservative structural analysis.

\section{Conclusion}

The mechanical design for the CCT quadrupole magnet prototype has been done. For stresses analysis, a periodic axial symmetry model related to two-layer CCT magnet with one pitch is taken to be solved in ANSYS. The loads by thermal contraction during cooling and Lorentz force are considered in the calculation. The results show that the displacement of the coil is very small at the effect of Lorentz force which is about 0.05 microns. The equivalent stress is less than $10 \mathrm{MPa}$ which indicates that the Lorentz force is separated by the ribs. And, the effect of Lorentz force on the result of stresses is quite weaker than the thermal strain. The results of the two analysis steps are almost same and the displacement and stress of the structure are dominant by thermal contraction. The maximum stress on the mandrels is about $300 \mathrm{MPa}$ which is within the allowable value.

\section{Data Availability}

The data used to support the findings of this study are available from the corresponding author upon request.

\section{Conflicts of Interest}

The authors have no conflicts of interest.

\section{Acknowledgments}

This study was supported by National Natural Science Foundation of China (91026004).

\section{References}

[1] W. L. Zhan and H. Xu, "Advanced fission energy programADS transmutation system," Bulletin of the Chinese Academy of Sciences, vol. 27, no. 3, pp. 375-381, 2012.

[2] J. C. Yang, J. W. Xia, G. Q. Xiao et al., "High intensity heavy ion accelerator facility (HIAF) in China," Nuclear Instruments and Methods in Physics Research Section B: Beam Interactions with Materials and Atoms, vol. 317, pp. 263-265, 2013.

[3] W. Wu, Y. Liang, W. Wu et al., "Multipole magnets for the HIAF fragment separator using the Canted-Cosine-Theta (CCT) geometry," Journal of Physics Conference Series, vol. 1401, 2018.

[4] Y. Liang, R\&D of the Canted-Cosine-Theta Superconducting Magnets, University of Chinese Academy of Sciences, Beijin, China, 2018.

[5] A. Hafalia, S. Caspi, H. Felice, L. Brouwer, S. Prestemon, and A. Godeke, "The structural design for a "canted cosine-theta" superconducting dipole coil and magnet structure-CCT1," IEEE Transactions on Applied Superconductivity, vol. 24, no. 3, pp. 1-4, 2014.

[6] Y. Ren, F. Wang, Z. Chen, and W. Chen, "Mechanical stability of superconducting magnet with epoxy-impregnated," Journal of Superconductivity and Novel Magnetism, vol. 23, no. 8, pp. 1589-1593, 2010.
[7] G. P. Willering, Stability of Superconducting Rutherford Cables: For Accelerator Magnets, University of Twente, Enschede, Netherlands, 2009.

[8] J. Fellinger, S. Freundt, D. Hathiramani, V. Bykov, and F. Schauer, "Dynamic response analysis of superconducting coils in wendelstein 7-X and mechanical quench test," Fusion Engineering and Design, vol. 86, no. 6-8, pp. 1385-1388, 2011.

[9] S. Caspi, F. Borgnolutti, L. Brouwer et al., "Canted-cosinetheta magnet (CCT)-A concept for high field accelerator magnets," IEEE Transactions on Applied Superconductivity, vol. 24, no. 3, pp. 1-4, 2014.

[10] L. Brouwer, S. Caspi, and S. Prestemon, "Structural analysis of an 18 T hybrid canted-cosine-theta superconducting dipole," IEEE Transactions on Applied Superconductivity, vol. 25, no. 3, pp. 1-4, 2015.

[11] S. Caspi, D. Arbelaez, L. Brouwer, S. Gourlay, S. Prestemon, and B. Auchmann, "Design of a canted-cosine-theta superconducting dipole magnet for future colliders," IEEE Transactions on Applied Superconductivity, vol. 27, no. 4, pp. 1-5, 2017.

[12] L. Brouwer, D. Arbelaez, S. Caspi, M. Marchevsky, and S. Prestemon, "Improved modeling of canted-cosine-theta magnets," IEEE Transactions on Applied Superconductivity, vol. 28, no. 3, pp. 1-6, 2018.

[13] L. Brouwer, D. Arbelaez, S. Caspi, H. Felice, S. Prestemon, and E. Rochepault, "Structural design and analysis of canted-cosine-theta dipoles," IEEE Transactions on Applied Superconductivity, vol. 24, no. 3, pp. 1-6, 2014.

[14] G. Montenero, B. Auchmann, L. Brouwer et al., "Mechanical structure for the PSI canted-cosine-theta (CCT) magnet program," IEEE Transactions on Applied Superconductivity, vol. 28 , no. 3, pp. 1-5, 2018.

[15] L. N. Brouwer, Canted-Cosine-Theta Superconducting Accelerator Magnets for High Energy Physics and Ion Beam Cancer Therapy, University of California, Berkeley, CA, USA, 2015.

[16] ANSYS Mechanical APDL, Release 19.0.

[17] Opera-3d Reference Manual, Version 15.0. 